\title{
Magnetic field amplification by high power lasers in underdense plasma
}

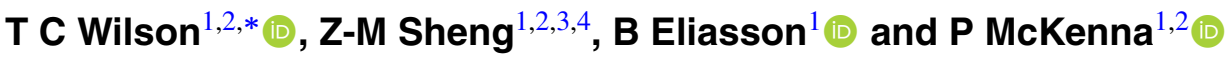 \\ ${ }^{1}$ SUPA, Department of Physics, University of Strathclyde, Glasgow G4 0NG, United Kingdom \\ ${ }^{2}$ Cockcroft Institute, Sci-Tech Daresbury, Cheshire WA4 4AD, United Kingdom \\ ${ }^{3}$ Key Laboratory for Laser Plasmas (MoE) and School of Physics and Astronomy, Shanghai Jiao Tong \\ University, Shanghai 200240, People's Republic of China \\ ${ }^{4}$ Collaborative Innovation Center of IFSA, Shanghai Jiao Tong University, Shanghai 200240, People's \\ Republic of China
}

E-mail: t.wilson@strath.ac.uk

Received 5 January 2021, revised 24 March 2021

Accepted for publication 14 April 2021

Published 8 June 2021

\begin{abstract}
The process by which an existing magnetic field of $\sim 10^{2}-10^{3} \mathrm{~T}$ may be amplified by an order of magnitude along the axis of laser propagation in underdense plasma by an intense laser pulse is investigated. The mechanism underlying the effect is understood to be ponderomotive in nature, initiated by the $\mathbf{E} \times \mathbf{B}$ drift motion of electrons displaced by the laser pulse as they relax towards the axis, and sustained by a combination of quasistatic magnetic field structures and electron Hall and diamagnetic currents. We employ two- and three-dimensional particle-in-cell simulations to numerically investigate the process and find qualitative agreement with the scaling relations found in our theory model. The lifetime of the process is considered, and we find the major factor limiting its growth and lifetime is ion motion, which disrupts the electron currents necessary to sustain the induced magnetic field. This field is found to be of sufficient strength, and is long-lived enough to be relevant for study in relation to applications in radiation production and laboratory astrophysics.
\end{abstract}

Supplementary material for this article is available online

Keywords: magnetised plasma, underdense plasma, short-pulse laser

(Some figures may appear in colour only in the online journal)

\section{Introduction}

Magnetic fields in plasmas are relevant to almost all laserplasma applications, as they are found universally in all plasma regimes from astrophysicsal to fusion and a need to understand them underpins many of the ongoing research efforts in plasma physics.

Very generally we may categorise magnetic fields as either self-generated within the plasma or externally imposed.

* Author to whom any correspondence should be addressed.

Original Content from this work may be used under the terms of the Creative Commons Attribution 4.0 licence. Any further distribution of this work must maintain attribution to the author(s) and the title of the work, journal citation and DOI.
A great variety of self-generated magnetic fields in laserproduced plasmas may be attributed to direct laser acceleration [1], often thermal and density gradients [2] and highintensity laser irradiation of solids may produce extremely strong fields into the tens of kilotesla [3]. Electrons accelerated by the laser may also produce magnetic fields via the inverse-Faraday effect [4-6] and more exotic schemes using Laguerre-Gaussian (LG) pulses [7]. Self-generated fields vary naturally in strength, topology and timescale over many orders of magnitude, and exploiting or mitigating their presence can be very challenging.

In contrast to this, external magnetic fields may be more precisely controlled, and imposed upon a plasma to induce specific effects. Solid-state and conventional electromagnets may be employed up to the order of $100 \mathrm{~T}[8,9]$, higher 
field strengths on the order of $1 \mathrm{kT}$ may be reached by using capacitor-coil targets [10,11] and yet higher fields maybe achieved with so-called snail targets irradiated by intense lasers $[12,13]$. Such high field regimes offer access to new regimes of physics and applications under unprecedented conditions.

Amplifying an existing magnetic field by magnetic flux compression has been explored previously in the context of inertial confinement fusion [14], whereby a frozen-in seed magnetic field is amplified via capsule implosion. More recently, Wu and Wang [15] studied the case in which an intense Laguerre-Gaussian (LG) laser incident on an underdense plasma with a preexisting background magnetic field is found to induce a highly localised area of ultra-strong magnetic field aligned along the path of the laser. The initial explanation of this is attributed to the transfer of angular momentum from an $\mathrm{LG}_{01}$ mode laser to the plasma, facilitated by the external magnetic field. Since then, an alternate explanation has been provided [16] involving the ponderomotive expulsion of electrons and the $\mathbf{E} \times \mathbf{B}$ drift acting on the return current.

We investigate the use of a linearly polarised pulse in the fundamental Gaussian mode, providing no angular momentum of its own for magnetic field amplification. In the presence of a background magnetic field, we find that the plasma nevertheless gains a significant amount of angular momentum, and a strong, static magnetic field forms in the wake of the pulse. We then study the characteristics and scaling relations for this induced field and examine the effect of ion motion. A theoretical model is provided to explain the magnetic field generation qualitatively.

\section{Simulation results}

In order to quantitatively understand the magnetic field amplification process, simulations were carried out using both the 3D Cartesian PIC code Osiris [17, 18] and the 2D FourierBessel PIC code FBPIC [19, 20]. Osiris is used to verify the principle and investigate the effect of ion motion, and FBPIC is used to perform parameter variations in order to better understand the process by which the magnetic field forms and is sustained. Characterising quantities in relation to the laser wavelength $\lambda_{0}$, the Osiris simulations used a static simulation box of size $40 \lambda_{0} \times 40 \lambda_{0} \times 40 \lambda_{0}$ with a resolution of 32 cells per laser wavelength in all directions, and 60 time steps per laser period $\tau_{0}=\lambda_{0} / c$. A preionised plasma is situated between $5 \lambda_{0} \leq z \leq 35 \lambda_{0}$ and extends to the transverse boundaries, leaving $1 \lambda_{0}$ of vacuum at the edges. There is 1 particle per species per cell, using quartic particle shapes. A linearly polarised laser with frequency $\omega$ and an initially Gaussian amplitude envelope $a=a_{0} \exp \left[-\left(r / R_{0}\right)^{2}-\right.$ $\left.\left(t / D_{0}\right)^{2}\right]$, with duration $D_{0}=10 \tau_{0}$, spot size $R_{0}=7 \lambda_{0}$ and peak amplitude of $a_{0}=5$ is incident on a uniform plasma slab with $n_{0}=n_{\mathrm{e}} / n_{\mathrm{c}}=0.5$ and a normalised background magnetic field of $B_{0}=\omega_{\mathrm{c}} / \omega=0.2$ directed along the $z$ axis, where $n_{\mathrm{c}}=$ $\omega^{2} \epsilon_{0} m_{\mathrm{e}} / e^{2}$ is the critical density, $\omega_{\mathrm{c}}=e B / m_{\mathrm{e}}$ is the electron cyclotron frequency and $\omega=2 \pi c / \lambda_{0}$ is the laser frequency. The FBPIC simulations use an similarly sized plasma to that

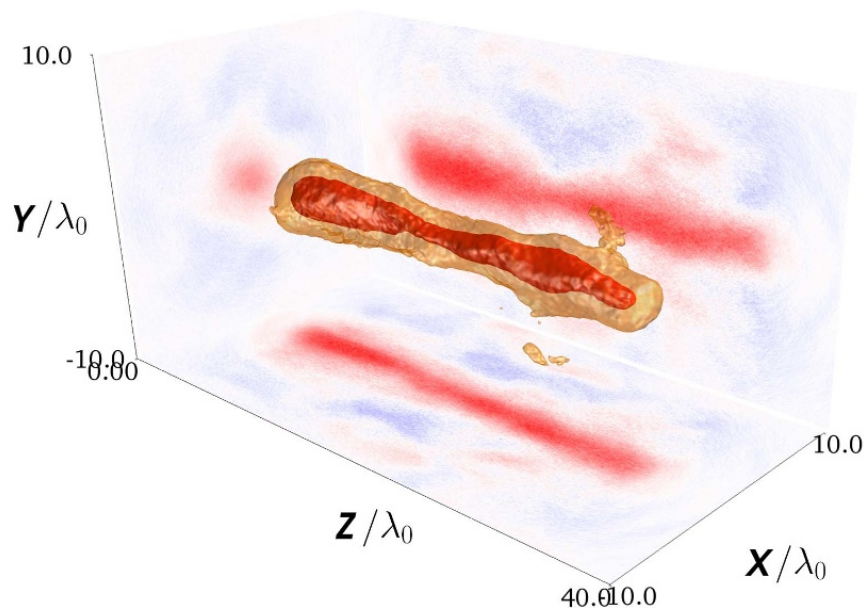

Figure 1. The $B_{z}$ field at $t=150 \tau_{0}$. The yellow isosurface corresponds to $B_{z}=0.2$, the red isosurface to $B_{z}=0.5$. The panels show projections of the $B_{z}$ field with blue indicating a reversal of sign of $B_{z}$. The peak field amplitude in the snapshot is $B_{z}=1.02$, corresponding to over $10^{4} \mathrm{~T}$ for $\lambda_{0}=1 \mu \mathrm{m}$. Reproduced with permission from [22].

of the Osiris simulations (beginning at $z=5 \lambda_{0}$, extending for $30 \lambda_{0}$ along $z$ and out to $r=19 \lambda_{0}$ ), but with a lower resolution of $16 \times 16$ cells $/ \lambda_{0}^{2}$, and where five azimuthal modes are used with $2 \times 2 \times 25$ particles per cell along $r, z, \theta$. We choose to use a cylindrical code for the parameter scans rather than a $2 \mathrm{D}$ code in order to better reproduce the 3D current structures that form, and we find that there is sufficiently good agreement between Osiris and FBPIC to justify its use [21]. The overall result is reproduced well, and the flexibility of a cylindrical code lends itself well to parameter scans. The cylindrical algorithm of FBPIC can result in noise close to the axis, however this can be compensated for with a slight smoothing filter, and instances where this technique is used are noted.

Osiris simulations are first carried out to reproduce the basic principle. A 3D render of the induced field is shown in figure 1. The ions are kept immobile and fixed in space. The very well defined cylindrical shape is due to the background magnetic field, which restricts particle motion perpendicular to the axis, but allows for particles to stream freely along $z$, allowing the field to spread to the edges of the plasma at $z=5$ and $z=35$. Examining a thin slice of the current density does not show any particularly strong azimuthal component, however when the current density is summed along $z$ for the whole simulation box, the azimuthal current becomes quite evident, as seen in figure 2. The lack of a visible azimuthal current in the slice diagnostics reinforces that the magnetic field is sustained by an averaged current.

The plasma beta is the ratio between the thermal pressure $P_{\mathrm{T}}=n_{\mathrm{e}} k_{\mathrm{B}} T$ and magnetic pressure $P_{\mathrm{B}}=B^{2} / 2 \mu_{0}$, given by

$$
\beta=\frac{P_{\mathrm{T}}}{P_{\mathrm{B}}}=\frac{n_{\mathrm{e}} k_{\mathrm{B}} T_{\mathrm{e}}}{B^{2} / 2 \mu_{0}},
$$

and is an important parameter to illustrate the energy transfer between plasma and magnetic fields. In order to analyse the 

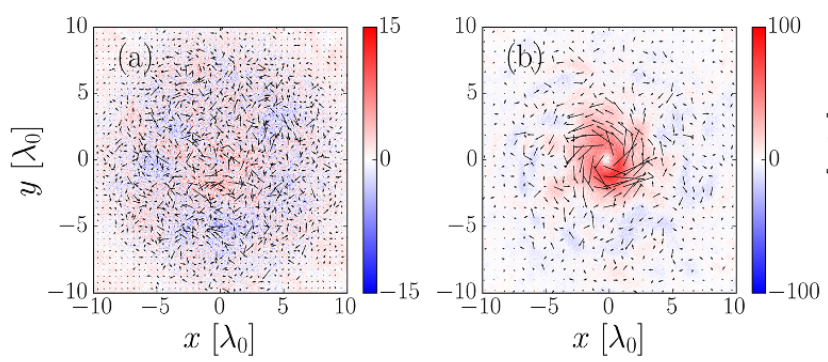

Figure 2. Azimuthal current density, at (a) a single slice through the middle of the simulation box (b) integrated over $z$. The quiver plots show the relative transverse current density vector at different points, being relatively incoherent in (a), and exhibiting azimuthal structure in (b). Reproduced with permission from [22].

plasma beta, an FBPIC simulation was performed. To improve resolution for electron temperature measurement, the number of azimuthal modes was reduced to 2 , and the number of particles per cell increased to 500. This produces an unphysically symmetric result, however the total energy, spatial extent, and time evolution of both the laser and amplified magnetic fields remain in-line with the full-3D simulation, so the result may be used to illustrate the physics. The plasma temperature $T$ is calculated by binning particles along $z$ and $r$ at the same resolution as the simulation grid, and calculating the mean kinetic energy of the particles within each bin. This is then substituted into 1 alongside the the azimuthally averaged plasma density $n_{\mathrm{e}}$ and total magnetic field $B$, calculated from the simulation diagnostic in order to retrieve the plasma beta. Once the laser has passed and the amplified field is established, $\beta$ approaches the form shown in figure 3(b). The component pressures are shown in figure 3(a). Close to the axis the electron temperature increases to about $2 \mathrm{MeV}$, which leads to a local increase of the electron pressure and a diamagnetic current (cf (3) below) which by itself would lead to a local decrease of the magnetic field due to the balance between kinetic and magnetic pressures. However, there is a local depletion of the electron number density in the channel which leads to a strong radial electric field with a resulting Hall current due to the $\mathbf{E} \times \mathbf{B}$ drift that exceeds the diamagnetic current and leads to a maximum of the magnetic field at $r=0$ and a minimum of the plasma beta, dropping to around 4. Moving away from the axis, the magnetic field strength drops quickly, but the temperature, and hence pressure, falls less sharply, resulting in a large increase in $\beta$ in the region out to approximately the laser spot size. Further away still, both the temperature and the magnetic field drop, and the plasma beta falls once more. At the far edge of the plasma, the electron temperature is still high, reaching over $250 \mathrm{keV}$. This is due to the necessarily constrained nature of the simulation box which does not allow heat to realistically dissipate outwards.

\section{Parameter scans}

Figure 4 shows a summary of parameter scans performed using FBPIC, varying several key parameters and examining the effect on the resulting magnetic fields, keeping the ions immobile. The results are presented as lineouts averaged in

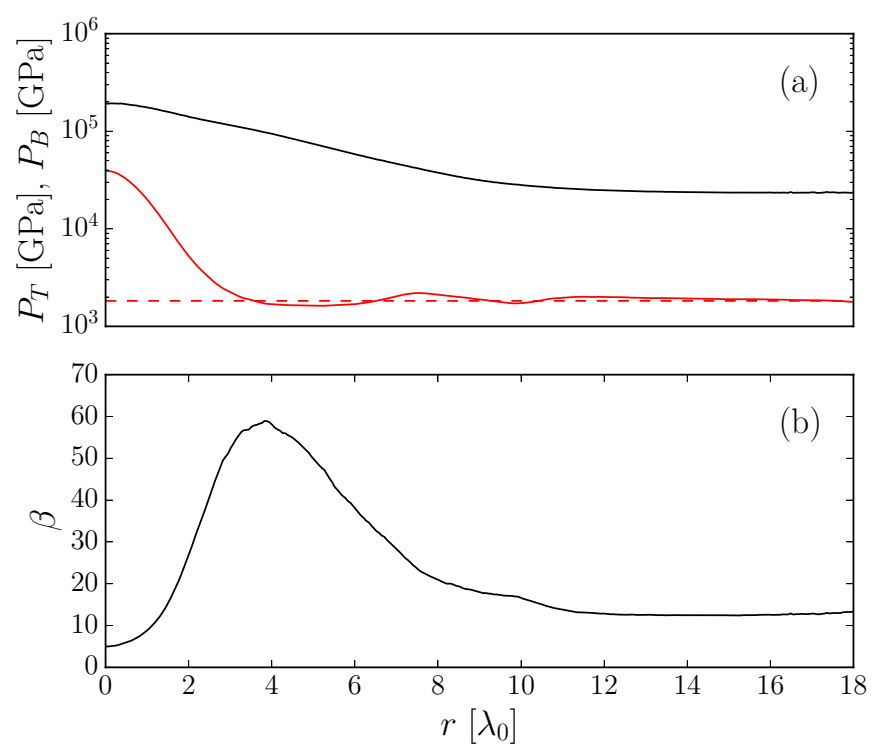

Figure 3. (a) The thermal (black) and magnetic (red) pressure. (b) The plasma beta. Both are averaged around $\theta$, and along $z$. Snapshot taken at $t=250 \tau_{0}$. The dashed red line corresponds to the baseline magnetic pressure from the seed magnetic field.

space around the azimuth and along the length of the plasma $5 \lambda_{0}<z<35 \lambda_{0}$, taken from the final timestep of the simulations at $t=250 \tau_{0}$. Spatial averaging is performed over both $z$ and $\theta$ to reduce the data to a $1 \mathrm{D}$ series in the radial direction only.

\subsection{Effect of laser parameters}

The electron temperature, momentum and current are related to the laser intensity, therefore we may intuitively expect to see field strength scaling with laser intensity. This notion is supported by figure 4(a), where the dotted line in the upper inset shows a good fit for $B^{2}$ as a function of $I$ to a linear regression. The lower inset shows the total energy of the $B_{z}$ field, which scales in a similar fashion.

Increasing the laser spot size increases the volume of the magnetic field as one might expect, demonstrated in figure $4(d)$. This is in line with the increased energy imparted to the plasma, as $\mathcal{E} \propto R_{0}^{2}$, so we also see a quadratic scaling of the magnetic field energy in the lower inset. The peak field strength however is not affected much, instead seeming to saturate, with the additional magnetic energy contained in the wider field profile. Very large spot-size beams are more prone to filamentation, so the beam does not focus onto the axis as cleanly as the smaller spot-size beams, As the simulated plasma is quite shallow and the beam itself already ultrashort, self correction and channel formation does not have time to occur and the laser energy is deposited over a large radius. This may also help to explain why, despite the increased energy imparted, the peak magnetic field does not increase as the beam cannot focus strongly enough to increase the amplitude on-axis much beyond the smaller spot-size simulations. 

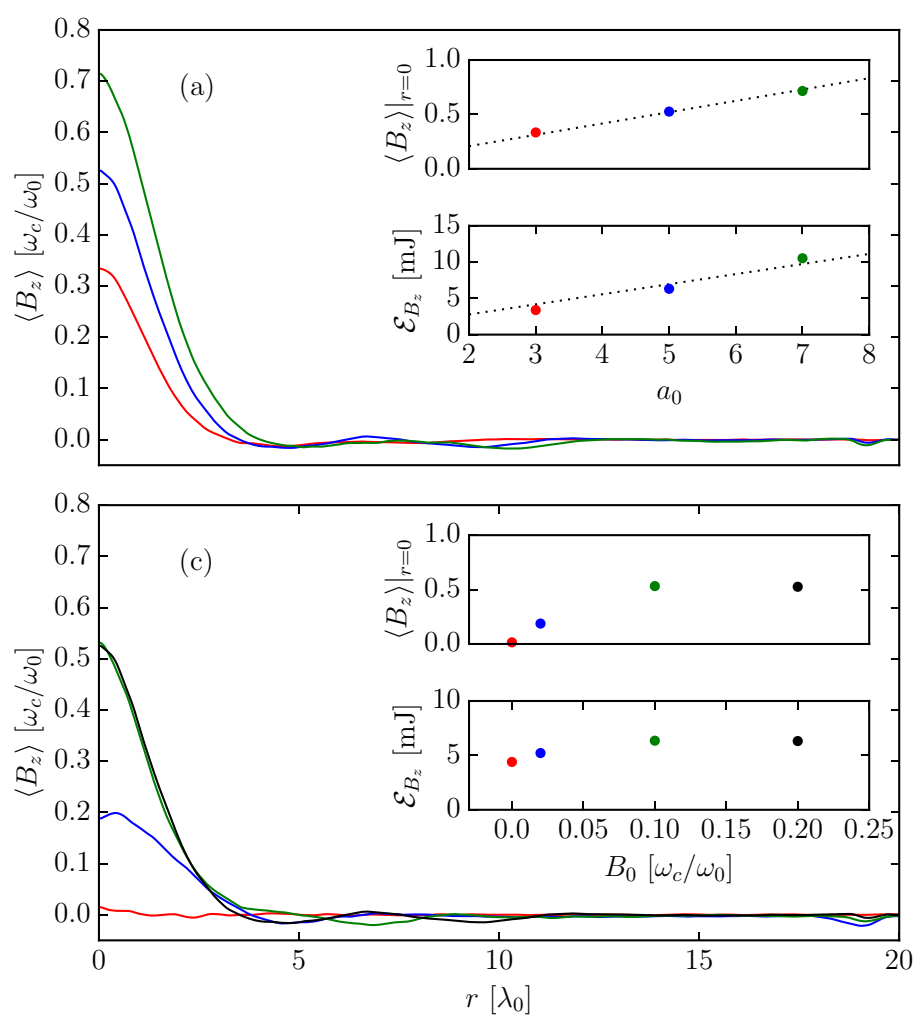
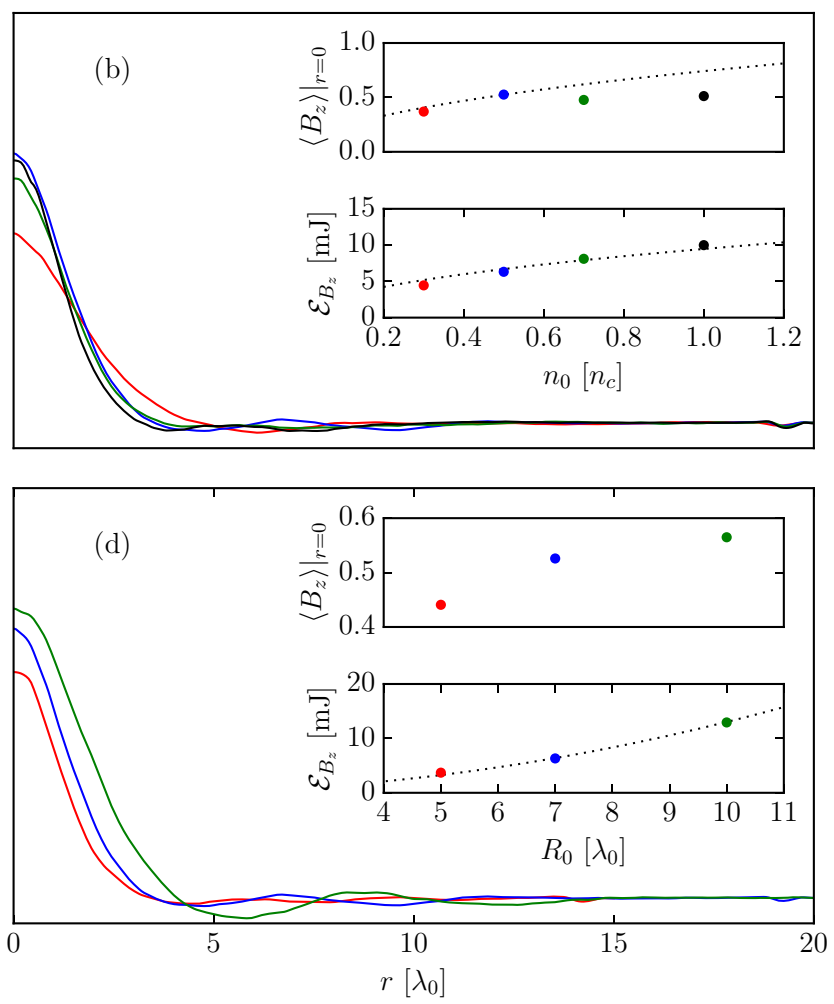

Figure 4. Radial profiles of the magnetic field obtained in 2D FBPIC simulations, demonstrating the effects of varying (a) laser intensity, (b) plasma density, (c) seed magnetic field strength and (d) laser spot size on $B_{z}$. All simulations use a common testbed with $B_{0}=0.2$, $a_{0}=5, R_{0}=7 \lambda_{0}, n_{0}=0.5 n_{\mathrm{c}}$, snapshots are taken at $t=250 \tau_{0}$ and spatially averaged around $\theta$ and along $z$. The averaged lineouts are smoothed with a Savitzky-Golay filter with a window length of 15 and polynomial of order 2 . The upper insets show the peak magnetic field (squared in the case of (a)) as a function of the parameter being varied. The lower insets show the total energy contained in the $B_{z}$ field. Both insets in (a) are fitted to a linear regression, both insets in (b) are fitted to a regression of power half. The lower inset in (d) is fitted to a quadratic regression. Reproduced with permission from [22].

\subsection{Effect of seed field strength}

The background field strength is more difficult to quantitatively examine, but nevertheless affects the induced field. The transverse heat flow is dependent on the magnetic field. A higher field restricts electron motion, leading to a sharper, tighter profile, and also changes the pitch angle of the current vortex running along the axis. Figure 4(c) shows the effect of varying $B_{0}$. Naturally when there is no initial magnetic field, no field is induced. However it is interesting to note from the lower inset that the energy contained in the $B_{z}$ field is not much less than the cases where an induced field is present. This suggests that the process does not greatly alter the magnetic energy partitioning, rather it simply reorders it to be more collimated. Further, the initial field has a saturation point around $B_{0}=0.1$ (corresponding to a real value of $\sim 1 \mathrm{kT}$ for $\lambda_{0}=1 \mu \mathrm{m}$ ), beyond which it no longer affects the induced field as seen in the upper inset. This may be explained as the point at which the majority of electrons close to the axis are contributing to the current. Beyond this, there is no further advantage to increasing the background field. This saturation point will increase linearly with laser amplitude as $p_{\perp} \propto a$, however the exact value is difficult to determine, relying on an accurate method to predict the residual momentum of the electrons after the laser has passed. This requires a knowledge of the specific focusing dynamics.

\subsection{Effect of plasma density}

The initial number density affects the energy coupling to the plasma, where increasing density with laser intensity remaining constant results in a lower temperature as the energy per particle is decreased. This relationship is tested with the results shown in figure 4(b). The averaged field amplitude appears to saturate after $n_{0}=0.5$, as illustrated in figure 4(b) upper inset. This is likely due to the aforementioned diminishing return on the energy coupling per particle for a constant laser intensity. Despite this, the total energy contained in the magnetic fields does increase with density, as shown in figure 4(b) upper inset. This may indicate that a high density plasma with $n_{0}$ $\geq 1$ is capable of supporting very high magnetic fields, but with the requirement that the laser pulse is of high enough intensity to sufficiently heat the plasma and drive the requisite currents.

The induced magnetic field forms quickly in the wake of the laser pulse, and appears to have an extremely long lifetime when the ions are immobile. In all cases the field persists until the simulation ends with very little change in topology, and only a minor decay in field strength. As the local plasma temperature can reach several hundred $\mathrm{keV}$ the collision frequency is expected to be negligible, as such, collisions were not simulated. The electron-ion collision rate can be approximated by [23] 


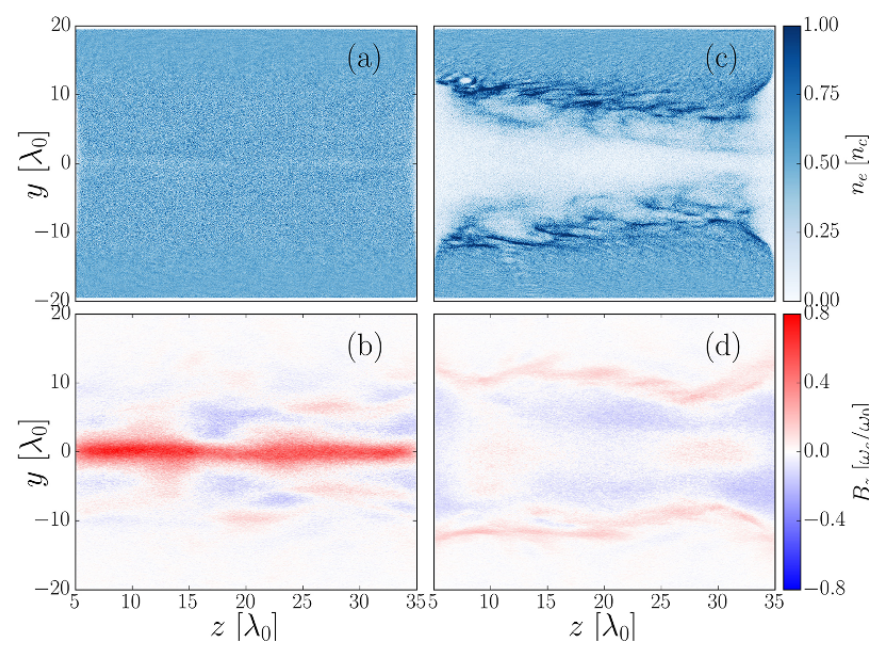

Figure 5. Comparison of electron densities (upper row) and $B_{z}$ field amplitudes (lower row) at $t=200 \tau_{0}$ in simulations without (a), (b) and with (c), (d) ion motion enabled. Reproduced with permission from [22].

$$
\nu_{\mathrm{ei}} \approx 2.91 \times 10^{-6} \mathrm{Zn}_{\mathrm{e}} T_{\mathrm{e}}^{-3 / 2} \ln (\Lambda)
$$

where $n_{\mathrm{e}}$ is given in $\mathrm{cm}^{-3}, Z$ is the number of free electrons per atom, $T_{\mathrm{e}}$ is the electron temperature in $\mathrm{eV}, \ln (\Lambda)=\ln \left(9 N_{\mathrm{D}} / Z\right)$ is the coulomb logarithm, $N_{\mathrm{D}}=4 \pi \lambda_{D}^{3} / 3$ is the volume of a Debye sphere and $\lambda_{\mathrm{D}}=\left(\epsilon_{0} T_{\mathrm{e}} / n_{\mathrm{e}} e^{2}\right)^{1 / 2}$ is the Debye length. A cursory estimate of the electron-ion collision frequency using the simulation plasma parameters, and taking an electron temperature of $100 \mathrm{keV}$ gives $\nu_{\mathrm{ei}} \sim 10^{9} \mathrm{~s}^{-1}$, corresponding to an potential timescale for the induced field on the order of nanoseconds, before collisions become significant. In future studies, it would be beneficial to study the effect collisions have on the energy transport, and determine quantitatively if there are significant losses to this mechanism.

\subsection{Effect of ion motion}

Despite the very high estimation of the field lifetime based on the collision frequency, ion motion substantially alters the process. Well before the onset of hydrodynamic expansion or collisional losses, we observe significant cavitation along the path of the laser. Figure 5 compares a simulation in which (hydrogen) ion motion is enabled to an otherwise identical simulation where it is not. With mobile ions, much of the areas close to the axis are evacuated shortly after the laser passes. As electrons move to neutralise the charge separation induced by the radial motion of the ions, this in turn disrupts the azimuthal current and leads to a rapid dissipation of the magnetic field. The induced field in this case is less collimated, with the onaxis strength much lower. However, there is still a relatively ordered field formed due to the fairly well-defined wall of the evacuated channel. As the channel expands, the radial motion of the plasma as a whole induces a complex expanding multiringed magnetic field structure, which may well be worthy of study in its own right. For experiment, the use of an ion species with a low charge-to-mass ratio, would delay the onset of this motion. This may be achieved with a high- $Z$ ion species.
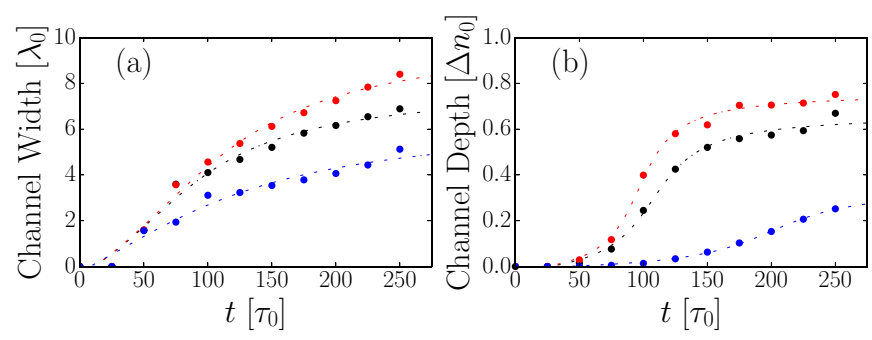

Figure 6. Ion channel formation rate for three ion species; $\mathrm{H}^{1+}$ (red), $\mathrm{Ar}^{18+}$ (black) and $\mathrm{Ar}^{1+}$ (blue) characterised by (a) channel width, defined as the radial point at which density is equal to the plasma plateau density, and (b) channel depth, defined as the density at $r=0$. The channel is not uniform, so a slice at $z=30 \lambda_{0}$ is chosen to illustrate the typical rates. The results are fitted to arctangent regressions, assuming the channel width and depth ultimately plateaus as ion momentum is disappated. Reproduced with permission from [22].

Figure 6 shows the rate of channel formation for three ion species, with realistic charge-to-mass ratios. Normalising charge to the electron charge $\tilde{q}=q / e$, and mass to the proton mass $\tilde{m}=m / m_{\mathrm{p}}$, we may compare the three cases. Hydrogen naturally has the highest normalised charge-to-mass ratio of 1 , resulting in the fastest channel growth. Fully ionised argon has a ratio of 0.45 , and singly ionised Argon has a ratio of 0.025 , and we observe the channel formation rate dropping along with the charge-to-mass ratio. The use of $\mathrm{Ar}^{1+}$ is illustrative, as the high laser fields and plasma temperature would ionise argon well beyond the first stage, but despite the much lower charge/mass ratio, a channel forms nonetheless. The effect of mixed-ion plasmas and partially ionised species are worthy of further study. Despite the drastic change in behaviour with mobile ions, the single-species simulations remain valuable to examine the physics involved in self-sustaining magnetic structures.

\section{Mechanisms}

\subsection{Qualitative explanation}

Both [15] and [16] offer differing explanations for the mechanism underlying the formation of the amplified field, the former attributing the angular momentum transfer to the plasma as coming from the laser, the latter from the external magnetic field. Certainly angular momentum transfer to the plasma electrons is vital to the formation and sustain of such a strong magnetic field, however the fact that the same effect may be observed using circular or even linearly polarised light suggests that the external magnetic field is the dominant source of angular momentum to the electrons rather than the laser itself. Considering the ponderomotive effect on electrons by the laser; electrons are expelled, and then when the peak of the laser has passed, they will begin to relax back towards the axis due to the resulting space charge electric field. As this happens, the external magnetic field bends the electron trajectories such that an azimuthal current is set up by the $\mathbf{E} \times \mathbf{B}$ drift of the electrons which seeds the eventual quasistatic magnetic field. 


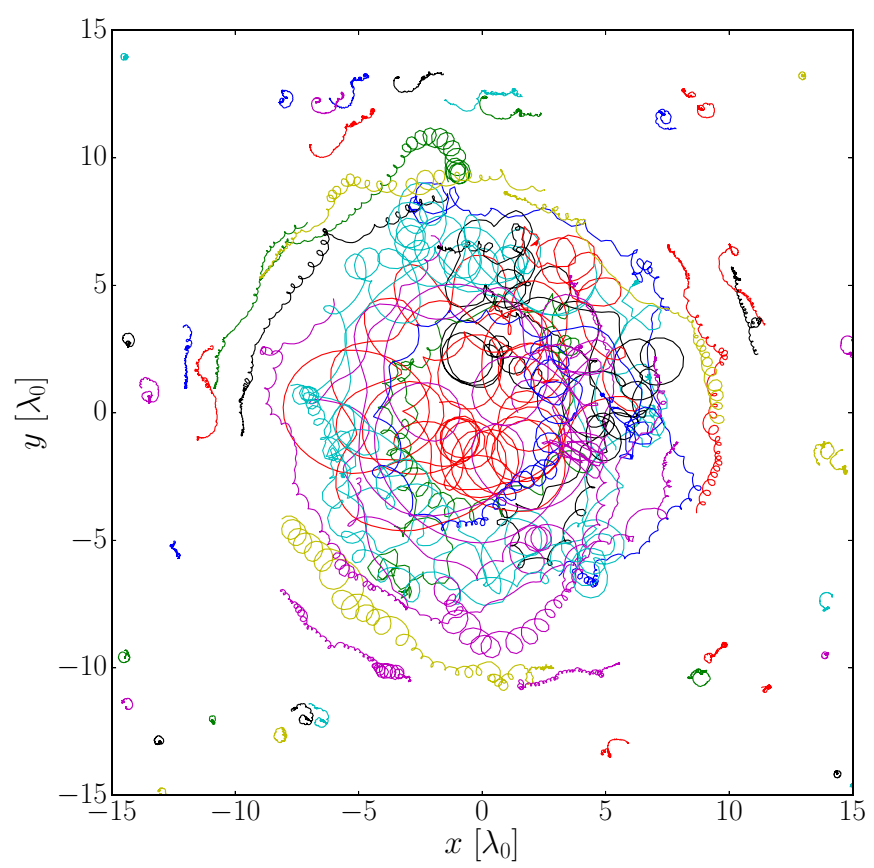

Figure 7. A flat random sample of 100 electron orbits. Spiral particle orbits are clearly seen, with the cyclotron rotation in the right-hand direction and the drift motion in the left-hand direction. Despite the higher magnetic field close to the axis, the Larmor radius $r_{\mathrm{L}}=m v / e B$ is larger due to that the electrons have been accelerated by the laser and gained speed in this region. Particle tracks are coloured arbitrarily to improve visibility. This figure is animated in the supplementary movie. Reproduced with permission from [22].

Once the field is established, it is then sustained via a continuous azimuthal current. In conjunction with this, we also consider the longitudinal motion of the electrons, and the resulting drift motion arising from guiding-centre motion of the electrons as the sustaining mechanism for the amplified field.

Assuming quasineutrality and neglecting ion motion, we may use Ampère's law to study the magnetic field including both drift motion and cyclotron motion

$$
\nabla \times \mathbf{B}=\mu_{0} \nabla \times \mathbf{M}_{\omega_{c}}+\mu_{0} \mathbf{J}_{\mathrm{f}}
$$

where $\mathbf{M}_{\omega_{c}}$ is the plasma magnetisation due to the cyclotron motion of the electrons and $\mathbf{J}_{\mathrm{f}}$ is a free current due to the grad$B$ drift of the electrons and the electron $\mathbf{E} \times \mathbf{B}$ drift due to the radial electric field set up charge separation. These electron motions are seen in figure 7 below. Thus, the right-hand side of (3) contains contributions both from the diamagnetic current [24] and the Hall current due to $\mathbf{E} \times \mathbf{B}$ drift. Due to plasma diamagnetism, any reactive magnetisation and drift currents will act to oppose an externally applied magnetic field. If we consider the effect of an intense laser on a plasma, ponderomotively accelerated electrons will form a negative axial current. This will in turn induce a negative azimuthal magnetic field. When coupled with the externally applied axial $B$-field, this combination sets up a twist in the magnetic field topology along the axis. Any electrons caught in this field structure will spiral along the field lines according to their $z$ momentum, and the pitch angle of the magnetic field vortex.
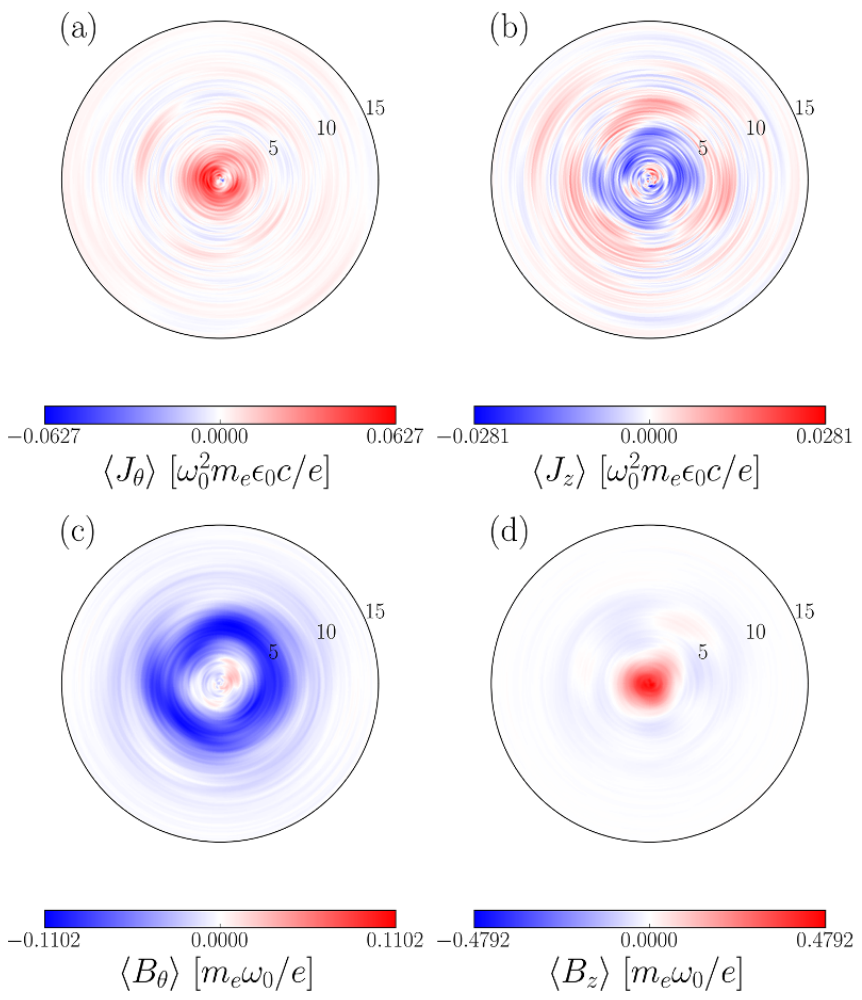

Figure 8. (a) $J_{\theta}$, (b) $J_{z}$, (c) $B_{\theta}$ and (d) $B_{z}$ field components at $t=200 \tau_{0}$, spatially averaged over the range $5 \lambda_{0}<z<35 \lambda_{0}$ illustrating the coupled azimuthal and axial fields required to sustain the axial $B$-field.

For a positive $B_{z}$ field, and a negative $B_{\theta}$ field, this translates to a twist in the $B$-field along $z$. Particles moving in the forwards $z$ direction acquire a negative rotational velocity, and hence produce a positive azimuthal current, which rather than acting diamagnetically as we might expect, in fact reinforces the axial $B$-field. Additionally, the sign of the external $B$-field is of no consequence, as reversing it also reverses the twist, and so a negative seed field may also be amplified via the same mechanism [15]. This behaviour can be seen in figure 7 and is animated in the supplementary movie (available online at stacks.iop.org/PPCF/63/084001/mmedia), where the transverse positions of a random sample of 100 electrons from the Osiris simulation are tracked, and the guiding centre motion can be clearly seen to oppose the cyclotron motion in almost all particles near to the laser axis. The configuration of currents and $B$-field components supporting this motion is illustrated in figure 8, extracted from a FBPIC simulation, which shows the equilibrium state of the simulated plasma long after the laser has passed. Despite the long timescale, the current and field structures remain well-defined with a net positive azimuthal current in (a), a net negative axial current in (b), a net negative azimuthal $B$-field in (c), and the compact, amplified axial $B$-field in (d). The seed $B$-field is not plotted.

Such well-defined quasistatic magnetic fields will naturally give rise to destructive drift motion in the plasma electrons, however at the timescale of our simulations, such motion appears insignificant when compared to the simple motion of forward-moving electrons spiraling along magnetic field lines. 


\subsection{Current amplification model}

In the following, we propose a theory model for transverse current amplification, which is responsible for magnetic field amplification. We temporarily revert back to dimensional units for $n_{0}$ and $B_{z}$ in this section. This is similar to the convectivecell model of current sheaths in space plasma [25]. Consistent with the FBPIC simulations in figure 4, we formulate this in one-dimensional Cartesian coordinates perpendicular to the magnetic field, which if we assume axial symmetry may be mapped to cylindrical geometry as $x \rightarrow r, y \rightarrow \theta$ and $z \rightarrow z$. An extension to cylindrical geometry is also possible, by using the conservation of energy and the $z$-component of the canonical angular momentum [26].

We assume that a short laser pulse propagating along a guiding magnetic field expels the electrons to create a wake with depleted electrons. Due to their larger mass, the ions initially do not move and the ion number density remains constant. The positive ion channel attracts the surrounding electrons by an electric field $\mathbf{E}$ pointing away from the channel. Due to the magnetic field the electrons perform $\mathbf{E} \times \mathbf{B}$ drift and sets up a perpendicular current that amplifies the magnetic field.

Electrons moving in a time-independent electrostatic field varying in the $x$-direction and time-independent magnetic field $B_{z}$ directed in the $z$-direction have two constants of motion $[27,28]$, the total energy

$$
\mathcal{E}=\frac{m_{\mathrm{e}}\left(v_{x}^{2}+v_{y}^{2}\right)}{2}-e \phi,
$$

and the canonical momentum

$$
P_{y}=m_{\mathrm{e}} v_{y}-e A_{y}
$$

which can be used to construct a time-independent solution to the non-relativistic Vlasov equation. The vector potential $A_{y}$ is related to the magnetic field $B_{z}$ as $\partial A_{y} / \partial x=B_{z}$. We assume that the ions form a homogeneous neutralizing background with density $n_{0}$. In general, time-independent solutions to the Vlasov equations are found on the form $f_{e}=f_{e}\left(\mathcal{E}, P_{y}\right)$. Here, the electron distribution function is chosen to be of the form

$$
f_{\mathrm{e}}\left(\mathcal{E}, P_{y}\right)=\frac{n_{0}}{2 \pi v_{T}^{2}} \exp \left[\frac{e \phi}{k_{\mathrm{B}} T_{e}}-\frac{v_{x}^{2}+v_{y}^{2}}{2 v_{T}^{2}}\right]\left[1-A_{e} \exp \left(\frac{\left(v_{y}-e A_{y} / m_{\mathrm{e}}\right)^{2}}{2 \alpha^{2} \omega_{c}^{2} \lambda_{D}^{2}}\right)\right]
$$

where $v_{\mathrm{T}}=\left(k_{\mathrm{B}} T_{\mathrm{e}} / m_{\mathrm{e}}\right)^{1 / 2}$ is the electron thermal speed of the Maxwellian distribution, $\lambda_{\mathrm{D}}=v_{\mathrm{T}} / \omega_{\mathrm{p}}$ is the electron Debye length, $\omega_{c}=e B_{0} / m_{\mathrm{e}}$ is the electron cyclotron frequency, $\omega_{p}=$ $\left(e^{2} n_{0} / \epsilon_{0} m_{\mathrm{e}}\right)^{1 / 2}$ is the electron plasma frequency, $\alpha$ determines the width of the channel (e.g. the beam width) in units of the Debye length, and $A_{\mathrm{e}}$ represents the amplitude, limited to $A_{\mathrm{e}} \leq 1$ for $f_{\mathrm{e}}$ to remain non-negative. The model (6) takes into account the local decrease of the electron number density but for simplicity neglects the electron temperature increase near the axis. The electron number density is given by

$$
n_{\mathrm{e}}=\int_{-\infty}^{\infty} \int_{-\infty}^{\infty} f_{\mathrm{e}} \mathrm{d} v_{x} \mathrm{~d} v_{y}
$$

The electron flux in the $y$-direction is

$$
n_{\mathrm{e}} v_{e y}=\int_{-\infty}^{\infty} \int_{-\infty}^{\infty} v_{y} f_{\mathrm{e}} \mathrm{d} v_{x} \mathrm{~d} v_{y}
$$

The vector potential is obtained from Ampère's law as

$$
\frac{\partial^{2} \tilde{A}_{y}}{\partial x^{2}}=\mu_{0} e n_{\mathrm{e}} v_{e y}
$$

and the scalar potential is governed by Poisson's equation

$$
\frac{\partial^{2} \phi}{\partial x^{2}}=\frac{e}{\epsilon_{0}}\left(n_{\mathrm{e}}-n_{0}\right)
$$

The vector and scalar potentials are nonlinearly coupled. At $x= \pm \infty$ we have $\partial A_{y} / \partial x=B_{z}=B_{0}$ so for convenience we set $A_{y}=\tilde{A}_{y}+B_{0} x$, and use the normalised, primed variables $x=x^{\prime} r_{\mathrm{D}}, \phi=\phi^{\prime} k_{\mathrm{B}} T_{\mathrm{e}} / e$ and $A_{y}=A_{y}^{\prime} B_{0} r_{\mathrm{D}}$ to obtain the dimensionless system

$$
\frac{\partial^{2} \tilde{A}_{y}^{\prime}}{\partial x^{\prime 2}}=-\frac{\beta_{T}^{2}}{\tilde{\omega}_{c}^{2}} \frac{A_{e} \alpha}{\left(\alpha^{2}+\tilde{\omega}_{c}^{-2}\right)^{3 / 2}} \mathrm{e}^{\phi^{\prime}}\left(\tilde{A}_{y}^{\prime}+x^{\prime}\right) \exp \left(-\frac{\left(\tilde{A}_{y}^{\prime}+x^{\prime}\right)^{2}}{2\left(\alpha^{2}+\tilde{\omega}_{c}^{-2}\right)}\right)
$$

$$
\frac{\partial^{2} \phi^{\prime}}{\partial x^{\prime 2}}=-1+\mathrm{e}^{\phi^{\prime}}\left[1-\frac{A_{e} \alpha}{\left(\alpha^{2}+\tilde{\omega}_{c}^{-2}\right)^{1 / 2}} \exp \left(-\frac{\left(\tilde{A}_{y}^{\prime}+x^{\prime}\right)^{2}}{2\left(\alpha^{2}+\tilde{\omega}_{c}^{-2}\right)}\right)\right]
$$

with boundary conditions $\tilde{A^{\prime}}{ }_{y}=0$ at $x^{\prime}=0$ and $\partial{\tilde{A^{\prime}}}_{y} / \partial x^{\prime}=0$ at $x^{\prime}=\infty$ for the vector potential, and $\partial \phi^{\prime} / \partial x^{\prime}=0$ at $x^{\prime}=0$ and $\phi^{\prime}=0$ at $x^{\prime}=\infty$ for the scalar potential. The system contains four dimensionless parameters: the amplitude $A_{\mathrm{e}}$, the width parameter $\alpha$, the cyclotron-to-plasma frequency ratio $\tilde{\omega}_{\mathrm{c}}=\omega_{\mathrm{c}} / \omega_{\mathrm{p}}$ and the normalised electron thermal speed $\beta_{\mathrm{T}}=$ $v_{\mathrm{T}} / c$. For $\alpha \ll \tilde{\omega}_{c}^{-1}$ the channel has a lower bound of the width as $\lambda_{\mathrm{D}} / \tilde{\omega}_{\mathrm{c}}=v_{\mathrm{T}} / \omega_{\mathrm{c}} \equiv \lambda_{\mathrm{L}}$, the thermal Larmor radius. 

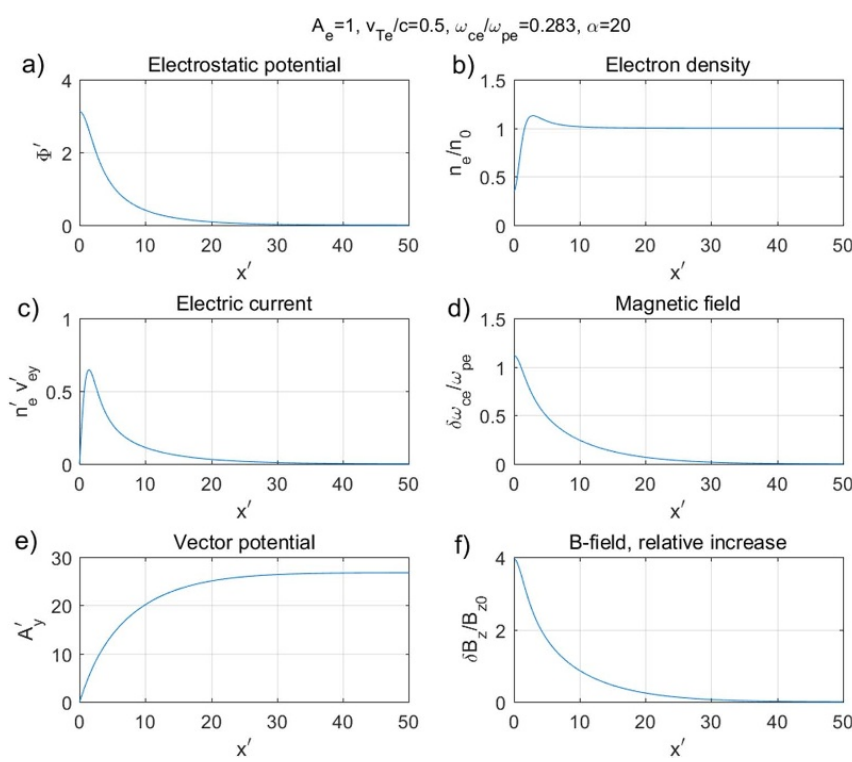

Figure 9. Spatial profiles of fields, for $A_{\mathrm{e}}=1, v_{\mathrm{T}} / c=0.5, \alpha=20$, and $\omega_{\mathrm{c}} / \omega_{\mathrm{p}}=0.283$. Here $x^{\prime}$ corresponds to $\left(r / \lambda_{0}\right) \lambda_{0} / \lambda_{\mathrm{D}}$ in figure 4 , where $\lambda_{0} / \lambda_{\mathrm{D}}=2 \pi\left(c / \nu_{\mathrm{T}}\right)\left(n_{\mathrm{e}} / n_{\mathrm{c}}\right)^{1 / 2} \approx 8.9$.

For weak magnetic fields such that $\omega_{\mathrm{p}}^{2} / \omega_{\mathrm{c}}^{2} \gg \alpha^{2}$ we have

$$
\frac{\partial^{2} \tilde{A}_{y}^{\prime}}{\partial x^{\prime 2}}=-A_{\mathrm{e}} \alpha \tilde{\omega}_{c} \beta_{\mathrm{T}}^{2} \mathrm{e}^{\phi^{\prime}}\left(\tilde{A}_{y}^{\prime}+x^{\prime}\right) \exp \left(-\frac{\tilde{\omega}_{\mathrm{c}}^{2}\left(\tilde{A}_{y}^{\prime}+x^{\prime}\right)^{2}}{2}\right),
$$

$$
\frac{\partial^{2} \phi^{\prime}}{\partial x^{\prime 2}}=-1+\mathrm{e}^{\phi^{\prime}}\left[1-A_{e} \alpha \tilde{\omega}_{\mathrm{c}} \exp \left(-\frac{\tilde{\omega}_{\mathrm{c}}^{2}\left(\tilde{A}_{y}^{\prime}+x^{\prime}\right)^{2}}{2}\right)\right],
$$

showing an increase of $\tilde{A}_{y}^{\prime}$ with increasing $\omega_{\mathrm{c}} / \omega_{\mathrm{p}}$. On the other hand, in the limit of a strong magnetic field $\omega_{\mathrm{p}}^{2} / \omega_{\mathrm{c}}^{2} \ll \alpha^{2}$ we have

$$
\begin{gathered}
\frac{\partial^{2} \tilde{A}_{y}^{\prime}}{\partial x^{\prime 2}}=-A_{\mathrm{e}} \frac{\beta_{T}^{2}}{\tilde{\omega}_{c}^{2} \alpha^{2}} \mathrm{e}^{\phi^{\prime}}\left(\tilde{A}_{y}^{\prime}+x^{\prime}\right) \exp \left(-\frac{\left(\tilde{A}_{y}^{\prime}+x^{\prime}\right)^{2}}{2 \alpha^{2}}\right), \\
\frac{\partial^{2} \phi^{\prime}}{\partial x^{\prime 2}}=-1+\mathrm{e}^{\phi^{\prime}}\left[1-A_{\mathrm{e}} \exp \left(-\frac{\left(\tilde{A}_{y}^{\prime}+x^{\prime}\right)^{2}}{2 \alpha^{2}}\right)\right],
\end{gathered}
$$

showing a decrease of $\tilde{A}_{y}^{\prime}$ with $\omega_{\mathrm{c}} / \omega_{\mathrm{p}}$.

Figure 9 shows the spatial profiles of the scalar and vector potentials, the electron number density, and magnetic field fluctuations for $A_{\mathrm{e}}=1, \beta_{\mathrm{T}}=0.5, \alpha=20$, and $\tilde{\omega}_{\mathrm{c}}=0.283$. The system is characterized by a local electron density depletion leading to a positive potential. The resulting electric field leads to $\mathbf{E} \times \mathbf{B}$ drift of the electrons in the $y$-direction, setting up an electric current that leads to an amplification of the magnetic field.

Figure 10 shows the dependence of the magnetic field increase $\delta \omega_{\mathrm{c}}$ at the centre of the channel, $x=0$, on the normalized guiding magnetic field strength $\omega_{\mathrm{c}} / \omega_{\mathrm{p}}$ and the normalized
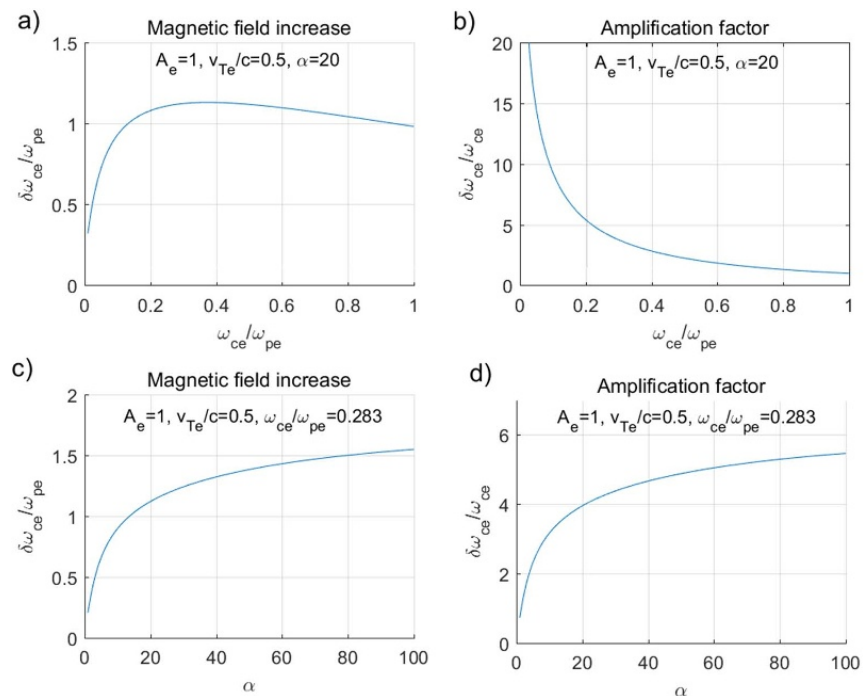

Figure 10. Top panels: magnetic field increase and amplification factor as a function of normalized magnetic field $\omega_{\mathrm{c}} / \omega_{\mathrm{p}}$, for $A_{\mathrm{e}}=1$, $v_{\mathrm{T}} / c=0.5$, and $\alpha=20$. Bottom panels: as a function of $\alpha$, for $A_{\mathrm{e}}=1, v_{\mathrm{T}} / c=0.5$, and $\omega_{\mathrm{c}} / \omega_{\mathrm{p}}=0.283$. Here $\delta \omega_{\mathrm{c}}=\tilde{B}^{\prime} \omega_{\mathrm{c}}$ represents the magnetic field increase.

channel width $\alpha$. As seen in figure 10(a), there is an optimal guiding magnetic field strength where the amplified magnetic field has a maximum. The electrons gyrate in the magnetic field with a typical radius equal to the electron thermal Larmor radius $r_{\mathrm{L}}=v_{\mathrm{T}} / \omega_{\mathrm{c}}$. For small values of the guiding magnetic field (or electron cyclotron frequency), the electron Larmor radius $r_{\mathrm{L}}$ will be comparable to or exceed the width of the channel so that the electrons gyrate in and out of the channel. In this case the electron $\mathbf{E} \times \mathbf{B}$ drift will be inefficient, and the resulting magnetic field amplification will decrease with decreasing guiding magnetic field $\left(\omega_{c} / \omega_{p}\right)$ as seen in figure 10 (a) for $\omega_{\mathrm{c}} / \omega_{\mathrm{p}}<0.2$, and consistent with equations (13) and (14). On the other hand, for a strong magnetic field where $r_{\mathrm{L}}$ is much smaller than the channel width, the electron $\mathbf{E} \times \mathbf{B}$ drift speed $v_{\mathrm{E}}=E / B_{z}$ will decrease for larger magnetic fields, leading to a decrease of the magnetic field amplification with increasing magnetic field, as can be seen in figure 10(a) for $\omega_{\mathrm{c}} / \omega_{\mathrm{p}}>0.5$, consistent with equations (15) and (16).

As seen in figures 10(c) and (d), the magnetic field amplification increases rapidly with the normalized channel width $\alpha$ (representing the laser beam radius in units of Debye lengths) for small values of $\alpha$ where the Larmor radius is comparable or smaller than the channel width. For large channel widths exceeding the Larmor radius the increase with channel width is relatively slow.

At later times when the ions start to move, they may also perform $\mathbf{E} \times \mathbf{B}$ drift, in the same direction as the electrons. This will lead to an ion current that will cancel the electron current, leading to decreased amplification of the magnetic field.

The profiles illustrated in figure 9 and the scaling shown in figure 10 qualitatively agree with our simulation results, which show scaling with input laser intensity $I_{0}$, initial plasma density $n_{0}$, initial magnetic field $B_{0}$, and laser spot size $R_{0}$. 
The magnetic field profile in figure 9(d) has a peak increase of the magnetic field of $\delta \omega_{\mathrm{ce}} / \omega_{\mathrm{pe}} \approx 1.1$ at $x^{\prime}=0$ which would correspond to a peak normalized magnetic field $\left\langle B_{z}\right\rangle \approx 0.78$ in figure 4 . The somewhat higher peak magnetic field in the theoretical model is attributed to diamagnetic drift by heated electrons near the center of the channel in the simulations (cf figure 8) which has been neglected in the theoretical model. The widths of the magnetic field profiles are roughly consistent between figure 9(d) and figure 4 . The dependence of the magnetic field increase on the background magnetic field in figure 10(a) is consistent with the simulation results in figure 4(c), where the increase is optimal at a certain background magnetic field but drops for lower magnetic fields due to the electron Larmor radius becoming comparable or larger than the channel radius, and also drops at higher background magnetic field due to the decrease of the $\mathbf{E} \times \mathbf{B}$ velocity and resulting Hall current. The magnetic field increase dependence on the width parameter $\alpha$ in figure 10(c) is also consistent with figure 4(d) where the magnetic field increase drops off rapidly at small channel widths and increases slowly at large widths.

The solutions to equations (11) and (12) rely on an assumed electron distribution function, and as such the result may be improved by incorporate the effect of the local electron heating in the choice of $f_{\mathrm{e}}$. Additionally, the theoretical model does not consider any longitudinal currents, which may also affect the result.

\section{Conclusions}

We have investigated the phenomena of magnetic field amplification by high power lasers, and demonstrated its viability for quasistatic magnetic fields lasting tens to hundreds of laser periods. The field is initially ponderomotively driven, allowing lasers of arbitrary polarisation and field configuration to be employed, given a suitable seed field of $\sim 500$ Tesla or higher. The field itself is sustained by an azimuthal current induced in the wake of the laser pulse.

The induced field scales with both laser amplitude and plasma density under the given parameters, with peak observed magnetic fields over $10 \mathrm{kT}$ and, sustained fields of 5-7 kT. Dense plasmas with ultraintense lasers may be capable of supporting fields further into the tens of kilotesla. It is interesting that while extremely strong magnetic fields may be produced, the overall energy contained within the relevant field component remains small when compared to the other components. This is likely due to the extremely compact nature of the field structure, but may suggest a way to improve the efficiency of the process and realise stronger fields given further research.

Due to the high power of the laser and high density of the plasma, self-focusing dominates the laser propagation as it passes through the plasma, focusing down to a thin filament within a few micrometers. This strong focusing and resulting ponderomotive expulsion is the initiator for the magnetic amplification. But also limits the effective range of the interaction. As the laser will tend to diffract after such strong focusing. In order to extend the interaction length, there may be some benefit to using a broader laser spot size, or shifting the plasma position relative to the focal plane, so that the beam is wider. Though beyond the scope of this work, it would be interesting to consider the additional instabilities this may introduce, such as filamentation. The amplification process relies, at least in part, on the symmetry of the system. Electrons are expelled purely radially, and return radially. In the event the beam is broken into multiple filaments, the ponderomotive force will become somewhat chaotic within the beam spot. This breaking of symmetry may give rise to entirely different current and field structures, possibly disrupting the effect.

Ion motion is destructive to the effect, as it disrupts the underlying electron current structures. Despite this, the amplified axial field still persists for hundreds of femtoseconds at least, making the phenomenon of useful duration nonetheless. The field lifetime may be further extended by the use of an ion species with a low charge-to-mass ratio. In such cases, it would be very useful to study the interactions of the amplification mechanism and other self-generated magnetic fields arising from laser-plasma interactions outlined in the introduction. Especially as high $\mathrm{Z}$ gas or $\mathrm{CH}$ targets may easily support near or over-critical densities, the surface magnetic fields may significantly affect the internal field structures.

We propose a kinetic theoretical model for current amplification and subsequent magnetic field amplification. It is found that a guiding magnetic field expels the electrons to create a plasma channel with depleted electrons. The positive ion channel attracts the surrounding electrons by an electric field pointing away from the channel. Due to the initially longitudinal magnetic fields, the electrons perform transverse drift motion and set up a perpendicular Hall current that amplifies the magnetic field. The model can qualitatively explain the simulation results.

Experimental benchmarking of this work would benefit from study of the methods employed in [10, 11, 13], as these experiments are closely related to this work, involving both strong magnetic fields and near-critical plasmas. The plasma density and magnetic fields may be diagnosed via proton deflectometry, polaro-inteferometry [29], Faraday rotation and $\mathrm{x}$-ray phase-contrast imaging.

\section{Data availability statement}

All data that support the findings of this study are included within the article (and any supplementary files).

\section{Acknowledgments}

The authors acknowledge the OSIRIS consortium, a United Kingdom Science and Technology Facilities Council Grant No. ST/P002056/1, and the Engineering and Physical Sciences Research Council (EPSRC) Grant No. EP/M009386/1. The numerical simulations were carried out on ARCHER via the Plasma HEC Consortium supported by EPSRC Grant Nos. EP/L000237/1 and EP/R029148/1. 


\section{ORCID iDs}

T C Wilson (D) https://orcid.org/0000-0002-6140-7045

B Eliasson (D) https://orcid.org/0000-0001-6039-1574

P McKenna (D) https://orcid.org/0000-0001-8061-7091

\section{References}

[1] Stamper J A 1991 Review on spontaneous magnetic fields in laser-produced plasmas: phenomena and measurements Laser Part. Beams 9841

[2] Biermann L 1950 Uber den usprung der magnetfelder auf sternen und im interstellaren raum Z. Naturforsch. 5a 65 (available at: https://zfn.mpdl.mpg.de/data/Reihe_A/5/ ZNA-1950-5a-0065.pdf)

[3] Wanger U et al 2004 Laboratory measurements of 0.7 GG magnetic fields generated during high-intensity laser interactions with dense plasmas Phys. Rev. E 70026401

[4] Sheng Z-M and Meyer-ter Vehn J 1996 Inverse Faraday effect and propagation of circularly polarized intense laser beams in plasmas Phys. Rev. E 541833

[5] Kostyukov I Y et al 2002 Magnetic-field generation and electron acceleration in relativistic laser channel Phys. Plasmas 9636

[6] Shvets G et al 2002 Magnetic field generation through angular momentum exchange between circularly polarized radiation and charged particles Phys. Rev. E 65046403

[7] Shi Y et al 2018 Magnetic field generation in plasma waves driven by copropagating intense twisted lasers Phys. Rev. Lett. 121145002

[8] Sims J R, Rickel D G, Swenson C A, Schillig J B and Ammerman C N 2008 Assembly, commissioning and operation of the NHMFL 100 Tesla multi-pulse magnet system IEEE Trans. Appl. Supercond. 18587

[9] Debray F and Frings P 2013 State of the art and developments of high field magnets at the 'Laboratoire National des Champs Magnétiques Intenses C. R. Phys. 142

[10] Fujioka $S$ et al 2013 Kilotesla magnetic field due to a capacitor-coil target driven by high power laser Sci. Rep. 31170

[11] Santos J J et al 2015 Laser-driven platform for generation and characterization of strong quasi-static magnetic fields $\mathrm{New}$ J. Phys. 17083051

[12] Korneev P, d'Humiéres E and Tikhonchuk V 2015 Gigagauss-scale quasistatic magnetic field generation in a snail-shaped target Phys. Rev. E 91043107
[13] Pisarczyk T et al 2018 Magnetized plasma implosion in a snail target driven by a moderate-intensity laser pulse Sci. Rep. 917895

[14] Gotchev O V et al 2009 Laser-driven magnetic-flux compression in high-energy-density plasmas Phys. Rev. Lett. 103215004

[15] Wu D and Wang J W 2017 Magetostatic amplifier with tunable maximum by twisted-light plasma interactions Plasma Phys. Control. Fusion 59095010

[16] Shi Y, Weichman K, Kingham R J, and Arefiev A V 2020 Magnetic field amplification in a laser-irradiated thin foil by return current electrons carrying orbital angular momentum (arXiv: 2001.06115)

[17] Fonseca R A et al 2002 OSIRIS, a three dimensional fully relativistic particle in cell code for modeling plasma based accelerators Lect. Notes Comput. Sci. 2331342

[18] OSIRIS I extreme plasma physics (available at: http:// epp.tecnico.ulisboa.pt/osiris/) (accessed 30 July 2020)

[19] Lehe R, Kirchen M, Andriyash I A, Godfrey B B and Vay J-L 2016 A spectral, quasi-cylindrical and dispersion-free particle-in-cell algorithm Comput. Phys. Commun. 20366

[20] FBPIC documentation (available at: https://fbpic.github.io/ index.html) (accessed 30 July 2020)

[21] Wilson T C et al 2019 Laser pulse compression towards collapse and beyond in plasma J. Phys. B: At. Mol. Phys. 52055403

[22] Wilson T C 2020 Control of nonlinear laser pulse propagation in plasma with strong magnetic fields $\mathrm{PhD}$ thesis University of Strathclyde

[23] Gibbon P 2005 Short Pulse Laser Interactions With Matter: An Introduction (London: Imperial College Press)

[24] Goldston R J and Rutherford P H 1995 Introduction to Plasma Physics (Bristol: IOP Publishing)

[25] Eliasson B and Shukla P K 2004 Localized kinetic structures in magnetized plasmas Phys. Scr. 200438

[26] Eliasson B and Shukla P K 2007 Theory for two-dimensional electron and ion Bernstein-Greene-Kruskal modes in a magnetized plasma J. Plasma Phys. 73715

[27] Harris E G 1962 On a plasma sheath separating regions of oppositely directed magnetic field Il Nuovo Cimento 23115

[28] Schindler K and Birn J 2002 Models of two-dimensional embedded thin current sheets from Vlasov theory $J$. Geophys. Res. 107 SMP 20-1

[29] Pisarczyk T et al 2015 Space-time resolved measurements of spontaneous magnetic fields in laser-produced plasma Phys. Plasmas 22102706 